\title{
PRODUÇÃO CIENTÍFICA BRASILEIRA SOBRE A PROMOÇÃO DA SAỨDE NA ATENÇÃO PRIMÁRIA: ESTUDO BIBLIOMÉTRICO
}

BRAZILIAN SCIENTIFIC PRODUCTION ON HEALTH PROMOTION IN PRIMARY CARE: BIBLIOMETRIC STUDY

PRODUCCIÓN CIENTÍFICA BRASILEÑA SOBRE PROMOCIÓN DE SALUd EN LA ATENCIÓN PRIMARIA: ESTUdIO BIBLIOMÉTRICO

Xavéle Braatz Petermann ${ }^{1}$

Sheila Kocourek ${ }^{2}$

\section{Como Citar:} Petermann XB, Kocourek S. Produção científica brasileira sobre a promoção da saúde na atenção primária: estudo bibliométrico. Sanare (Sobral, Online). 2021;20(1):81-90.

Palavras-chave: Promoção da Saúde; Atenção Primária à Saúde; Bibliometria.

Keywords: Health Promotion; Primary Health Care; Bibliometrics.

Palabras clave: Promoción de Salud; Atención Primaria a la Salud; Bibliometría.

Submetido: $16 / 05 / 2020$

Aprovado: $13 / 11 / 2020$

Autor(a) para Correspondência: Xavéle Braatz Petermann E-mail:xavelepetermann@gmail.com

\section{RESUMO}

Este estudo teve por objetivo analisar a produção científica sobre a promoção da saúde na atenção primária à saúde no contexto brasileiro. Para isso, realizou-se uma pesquisa bibliométrica com abordagem exploratóriodescritiva, por meio da busca de artigos na base de dados SciELO, no período de 2006 a novembro de 2019, com a combinação dos descritores "promoção da saúde and atenção primária à saúde" no resumo dos manuscritos, sendo incluídos 123 artigos. Os resultados evidenciaram o predomínio de artigos nos anos de 2017 e 2016, nas Revistas Ciência \& Saúde Coletiva e Saúde Pública, respectivamente. Relativo ao assunto do artigo relacionado à promoção da saúde na atenção primária, a maioria abordou a alimentação/ nutrição e assistência farmacêutica. Identificou-se também que o método empregado com maior frequência foi o qualitativo e a população de estudo mais frequente tratou-se dos profissionais de saúde e os idosos. Foi possivel obter um panorama da produção acadêmica nacional, de modo a direcionar, ainda, as lacunas existentes na produção e consequente evolução da temática no período analisado.

\footnotetext{
1. Fisioterapeuta. Mestre em Gestão de Organizações Públicas pela Universidade Federal de Santa Maria (UFSM). Fisioterapeuta na Prefeitura Municipal de Arroio do Tigre, RS. E-mail: xavelepetermann@gmail.com. ORCID: https://orcid.org/0000-0003-2672-0164

2. Assistente Social. Doutora em Serviço Social pela Pontifícia Universidade Católica do Rio Grande do Sul (PUCRS). Docente na Universidade Federal de Santa Maria, RS. E-mail: sheilakocourek@gmail.com. ORCID: https://orcid. org/0000-0001-8962-8758
}

Cert. de Redação Científica: Central das Revisões. Edição de texto: Karina Maria M. Machado. Revisão de provas: Texto definitivo validado pelos(as) autores(as). 


\section{ABSTRACT}

This study aimed to analyze the scientific production on health promotion in primary health care in the Brazilian context. With this in mind, a bibliometric research was carried out with an exploratory-descriptive approach, by means of an article search in the SciELO database, from 2006 to November 2019, with the combination of the descriptors "Health Promotion" and "Primary Health Care" in the abstract of the manuscripts, with the inclusion of 123 articles. The results showed the predominance of articles in the years 2017 and 2016, in the journals Ciência \& Saúde Coletiva and Saúde Pública, respectively. Regarding the subject of the article related to health promotion in primary care, most addressed food/nutrition and pharmaceutical support. We also identified that the method most frequently used was qualitative, and the most frequent study population were health care professionals and elderly people. We managed to capture a panorama of the national academic production in order to address the gaps that still exist in the production and consequent evolution of the subject in the period analyzed.

\section{RESUMEN}

Este estudio tuvo como objetivo analizar la producción científica sobre la promoción de salud en la atención primaria a la salud en el contexto brasileño. Para eso, se realizó una investigación bibliométrica con abordaje exploratorio descriptiva, por medio de búsqueda de artículos en la base de datos SciELo, en el período de 2006 hasta noviembre de 2019, con el combinado de los descriptores "Promoção da Saúde" y "Atenção primaria à saúde" en el resumen de los manuscritos, siendo incluidos 123 artículos. Los resultados evidenciaron el predominio de artículos en los años de 2017 y 2016, en las Revistas Ciência \& Saúde Coletiva y Saúde Pública, respectivamente. Relativo al contenido del artículo relacionado a la promoción de salud en la atención primaria, la mayoría abordó la alimentación/nutrición y la asistencia farmacéutica. Se identificó también que el método empleado con más frecuencia fue el cualitativo y la población de estudio más frecuente fueron los profesionales de salud y los ancianos. Fue posible obtener un panorama de la producción academia nacional, de modo que direccione, además, los vacios existentes en la producción y consecuente evolución del tema en el período analizado.

\section{INTRODUÇÃO}

A promoção da saúde surge, principalmente, como reação à intensa medicalização da saúde, adotando a articulação de conhecimentos técnicos e populares para o enfrentamento do processo saúde-doença por meio de uma visão ampliada dos determinantes e condicionantes ${ }^{1}$. Como marco inicial, em 1974, Marc Lalonde publicou o Informe Lalonde, uma referência para o conceito de promoção da saúde. Nesse relatório, a saúde foi descrita como resultado da biologia, do meio ambiente, do modo de vida e da organização dos sistemas de saúde, sendo que os altos investimentos em assistência médica seriam insuficientes para atuar sobre os determinantes e condicionantes da saúde ${ }^{2}$.

0 impacto mais significativo do Informe Lalonde foi a I Conferência Internacional sobre Cuidados Primários de Saúde, realizada em Alma-Ata, na República do Cazaquistão, em 1978. A Declaração de Alma-Ata apresentou os primeiros consensos mundiais sobre o paradigma da promoção da saúde como item essencial das políticas públicas ${ }^{3}$.
A partir da Conferência supramencionada foram realizadas várias outras conferências para debater os temas relacionados à atenção primária e promoção da saúde. A Primeira Conferência Internacional sobre Promoção da Saúde foi realizada em Ottawa, Canadá, em 1986, e definiu a promoção da saúde como o processo de capacitação da comunidade para atuar na melhoria das suas condições de vida e saúde 4 .

Esse conceito é um marco para a promoção da saúde e, depois dessa Conferência, já foram realizadas oito iniciativas internacionais, que foram sediadas nas cidades de Adelaide (1988), Sundsvall (1991), Jacarta (1997), México (1999), Bangkok (2005), Nairóbi (2009), Helsinque (2013) e Xangai (2016). Em cada uma dessas conferências foram aprovadas declarações internacionais com enfoque na promoção da saúde, que buscaram atualizar os objetivos e as diretrizes descritas inicialmente em 0ttawa 5 .

No Brasil, as propostas e diretrizes das Conferências Internacionais sobre a promoção da 
saúde começam a se concretizar com o movimento da Reforma Sanitária nas décadas 70 e 80, tendo como marco a $8^{a}$ Conferência Nacional de Saúde (1986), a publicação da Constituição Federal em 1988 e a criação do Sistema Único de Saúde (SUS), de modo que, após 1994, foi implantada a Estratégia Saúde da Família, a qual atua de acordo com os princípios da promoção da saúde ${ }^{2}$.

Entretanto, a primeira iniciativa de promoção da saúde como política pública foi realizada em 2002, quando o Ministério da Saúde (MS) elaborou um documento para a discussão da Política Nacional de Promoção da Saúde (PNPS) ${ }^{6}$. Todavia, a PNPS somente foi aprovada em 2006, por meio da Portaria 687 GM/MS de 20067. 0 objetivo dessa política é promover a qualidade de vida, reduzir vulnerabilidades e riscos à saúde relacionados aos determinantes e condicionantes da saúde?

Em 2014, com o objetivo de aprimorar e atualizar a PNPS, foi lançada uma nova versão dessa política pública, apontando para os novos desafios do SUS e reconhecendo que suas prioridades ainda estão vinculadas à versão anterior ${ }^{8}$. A PNPS revisada aponta para a necessidade da articulação com outras políticas, em especial a Política Nacional de Atenção Básica, para o fortalecimento da promoção da saúde, bem como da importância da participação social. Sendo assim, objetivos, princípios, valores, diretrizes, temas transversais, estratégias operacionais, responsabilidades e temas prioritários, reformulados e atualizados, visam à equidade, à melhoria das condições e dos modos de viver e à afirmação do direito à vida e à saúde ${ }^{8}$.

Malta et al. ${ }^{9}$ descrevem que a PNPS aponta para o imperativo de articulação com outras políticas públicas e com o controle social, em razão da impossibilidade do setor saúde responder individualmente às condições de saúde da população. Complementando, Dias et al. ${ }^{10}$ relataram a importância de ações de promoção da saúde voltadas para a diminuição das iniquidades sociais, objetivando o fortalecimento da ação comunitária e a valorização do conhecimento popular, considerados bases da promoção da saúde.

Este estudo, ao considerar a temática da promoção da saúde na atenção primária como um tema relevante, tanto no campo empírico quanto teórico, voltou-se para compreender alguns aspectos das publicações científicas nacionais por meio de um estudo bibliométrico. A promoção da saúde é base para as práticas profissionais na atenção primária, sobretudo no contexto brasileiro, por meio do reconhecimento da importância da promoção da saúde na melhoria das condições de saúde da comunidade. Sendo assim, este artigo teve por objetivo analisar a produção científica sobre a promoção da saúde na atenção primária à saúde, no contexto brasileiro, na base de dados Scientific Electronic Library Online (SciELO).

\section{METODOLOGIA}

0 estudo realizado se caracteriza como uma análise bibliométrica de caráter exploratóriodescritivo. A bibliometria se caracteriza por estudos que utilizam métodos estatísticos e matemáticos na análise de textos científicos, em determinada área do conhecimento ${ }^{11}$. A importância de pesquisas bibliométricas foi reconhecida como uma metodologia de estudo que utiliza procedimentos específicos para a identificação e mapeamento de temáticas ainda pouco exploradas no meio científico ${ }^{12}$, sendo usada na área da saúde para identificar as tendências e evidências de pesquisa ${ }^{13}$.

Para realizar o estudo, optou-se por utilizar a SciEl0, base de dados em forma de biblioteca eletrônica que abrange uma coleção selecionada de periódicos científicos brasileiros, tendo por objetivo a preparação, armazenamento, disseminação e avaliação da produção científica ${ }^{14}$.

$\mathrm{Na}$ pesquisa realizada na base SciELO, utilizouse como associação os Descritores em Ciências da Saúde (DeCS): "promoção da saúde and atenção primária à saúde", de modo que essas palavras deveriam estar no resumo do manuscrito. A busca retornou 252 documentos, dos quais foram incluídos apenas artigos realizados no Brasil, da área da Ciência da Saúde e publicados no período de 2006 a novembro de 2019. A aplicação dos filtros descritos acima permitiu afunilar os resultados de forma automática, sendo que a escolha dos filtros foi baseada no objetivo do estudo. Desse modo, foram selecionados 123 estudos. Justifica-se a utilização do ano de 2006 por ser o ano da publicação da primeira versão da PNPS.

Para a compilação dos dados foi criada uma planilha no software Microsoft Excel ${ }^{\circledR 2007, ~ a ~}$ fim de proporcionar a identificação, registro e armazenamento das informações de cada artigo. $\mathrm{Na}$ planilha foram elencados os seguintes dados de 
coleta: título, ano de publicação, periódico, autores do artigo, palavras-chave (tema principal) e método. Extraídas as informações bibliométricas para a análise dos dados, procedeu-se à compilação quantitativa e posterior transformação em dados visuais.

\section{RESULTADOS E DISCUSSÃO}

Referente às publicações por período sobre a temática da promoção da saúde na atenção primária (Figura 1), o maior número de publicações foi observado nos anos de $2017(n=24)$ e $2016(n=16)$, em razão da revisão da PNPS em 2014, fomentando o debate e a publicação sobre o tema no contexto brasileiro. Nota-se também que o número de publicações começou a aumentar apenas em 2011, cinco anos após a publicação da primeira versão da PNPS.

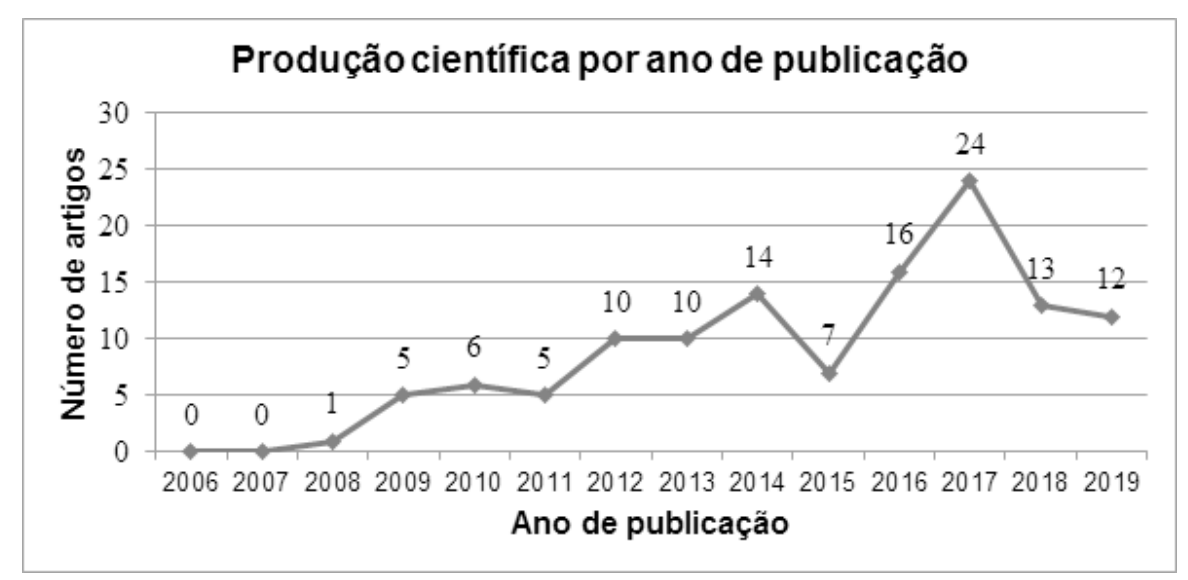

Figura 1. Linha do tempo da produção científica $(n=123)$.

Fonte: Dados da pesquisa.

Esses achados indicam a necessidade de pesquisas sobre a temática nos serviços de atenção primária. Paim ${ }^{15}$ afirma que, no contexto do SUS, ainda existe uma forte reprodução do modelo centrado na doença, na reabilitação e nos serviços especializados, em detrimento das ações de promoção da saúde na comunidade e no nível primário.

Prado et al. ${ }^{16}$ apontam para a necessidade do debate e da problematização sobre os percursos, as implicações, as possibilidades e as perspectivas para a consolidação das ações de promoção da saúde no contexto da atenção primária. Ao mesmo tempo, Malta et al. ${ }^{17}$ destacam que após 30 anos da criação do SUS ainda existe o imperativo de superar o modelo de cuidado centrado na doença. 0s mesmos autores ${ }^{17}$ refletem sobre o papel da PNPS, em especial, ao se considerar estratégias para o enfrentamento dos desafios impostos pelo perfil demográfico e epidemiológico da população.

Em seguida, a Figura 2 apresenta o número de artigos por periódicos; a partir disso, nota-se que a fonte líder para o tema é a Revista Ciência \& Saúde Coletiva, com 19 publicações, seguida da Revista de Saúde Pública, com 13 publicações. A Revista Ciência \& Saúde Coletiva foi criada em 1996, sendo editada pela Associação Brasileira de Saúde Coletiva (ABRASCO), caracterizando-se como um espaço científico para discussões, debates, apresentações de pesquisas, exposição de novas ideias e de controvérsias sobre a área de Saúde Coletiva ${ }^{18}$. A Revista de Saúde Pública, segunda colocada em número de publicações, foi criada em 1967, publicada sob responsabilidade da Faculdade de Saúde Pública da Universidade de São Paulo, descrita como um periódico inter e multidisciplinar que publica trabalhos científicos relevantes para a Saúde Pública no Brasil ${ }^{19}$. 


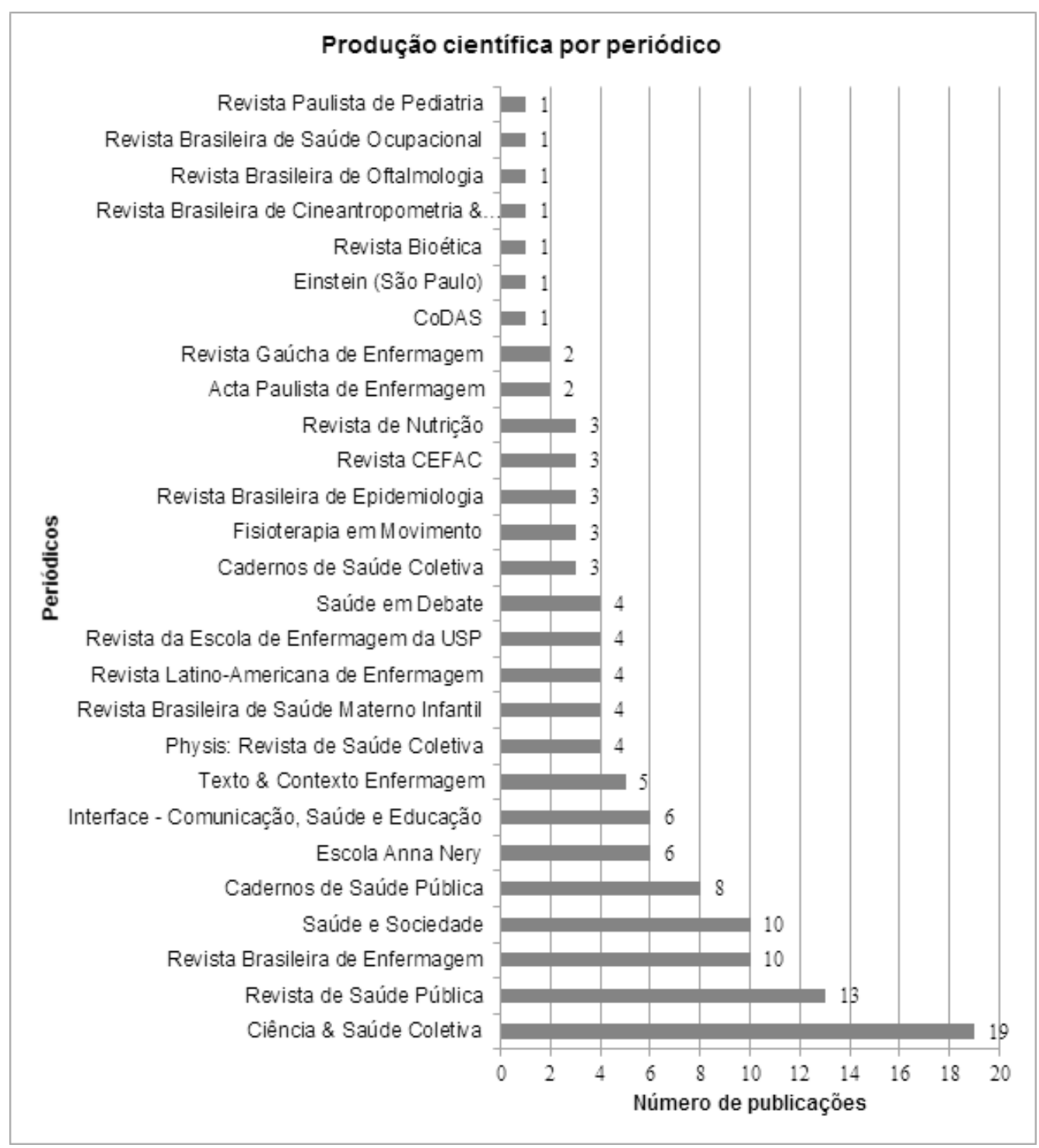

Figura 2. Produção científica por periódico $(n=123)$.

Fonte: Dados da pesquisa.

No que se refere ao assunto dos artigos (Figura 3) relacionado à promoção da saúde na atenção primária, foi possivel constatar, por meio das palavras-chave e, em alguns casos, dos títulos e subtítulos dos estudos, que 11 deles tinham como tema principal a alimentação e nutrição, enquanto dez artigos abordaram o tema da assistência farmacêutica. Salienta-se que foram diversos os assuntos relacionados ao tema da promoção da saúde na atenção primária, devido ao caráter intersetorial, interdisciplinar e multidisciplinar da temática investigada. 


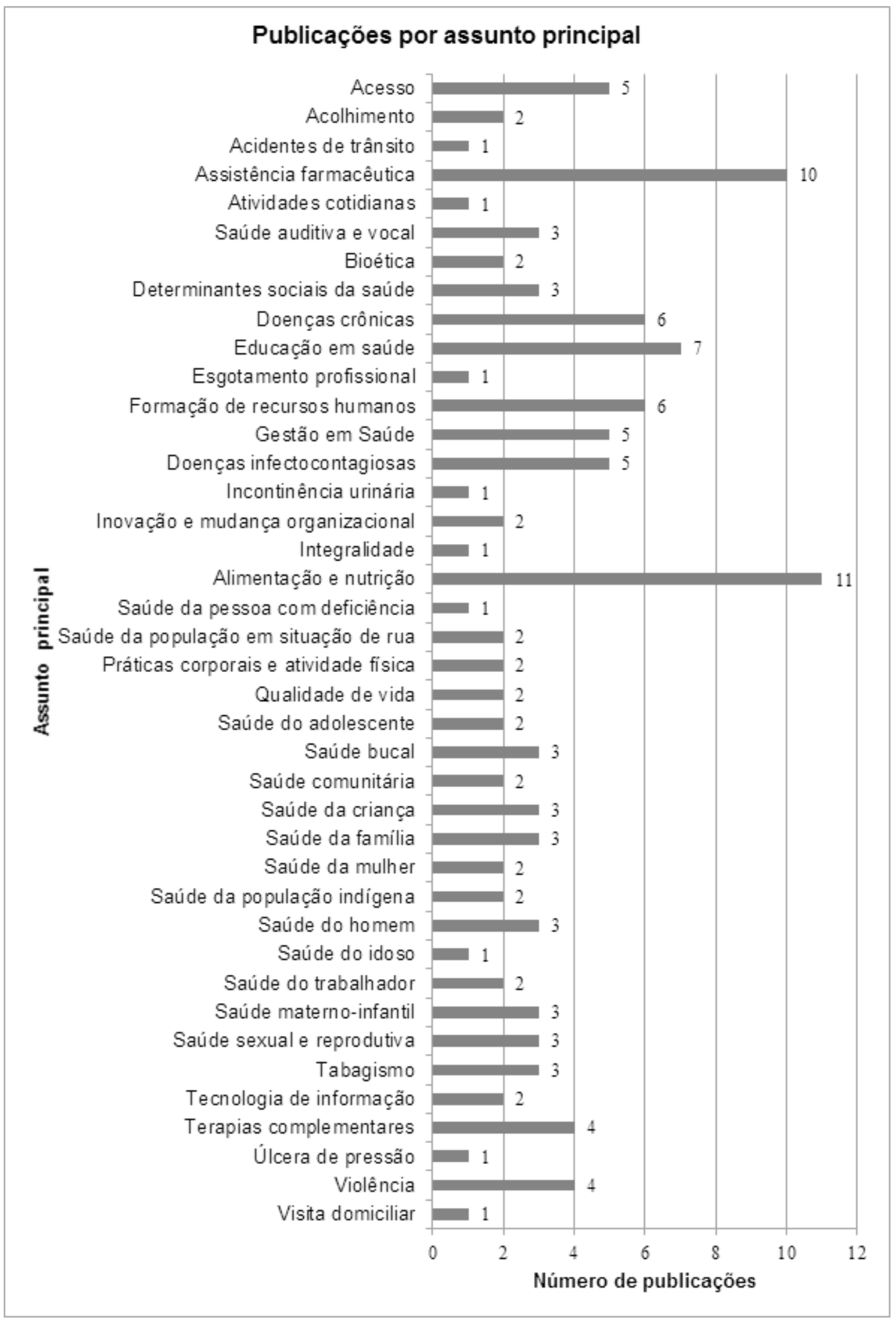

Figura 3. Número de publicações por assunto principal $(n=123)$.

Fonte: Dados da pesquisa (2019).

Cabe ressaltar que o tema da alimentação e nutrição está descrito na PNPS7 como um dos temas prioritários para a promoção da saúde, tendo como eixos norteadores a alimentação saudável e adequada e a segurança alimentar e nutricional, contribuindo com a diminuição da pobreza e garantia do direito à alimentação adequada 
e saudável. Corroborando o que foi descrito, Jaime et al. $^{20}$ descrevem que a área de alimentação e nutrição possui uma íntima relação com a promoção da saúde, entretanto os avanços no contexto brasileiro na diminuição da fome e desnutrição, aliados à transição nutricional, colocam novos e complexos desafios ao sistema público de saúde e à promoção da alimentação saudável e adequada.

Complementando, a PNPS buscou contribuir para a educação em saúde com base na alimentação e nutrição, adesão a rotinas alimentares mais saudáveis e implementação de ações que regulam a comercialização de alimentos nocivos ${ }^{21}$. Assim, a promoção da saúde seria um meio para transformar essas condições complexas em busca de melhores condições de saúde para a população, considerando os sujeitos como biopsicossociais.

Sobre a assistência farmacêutica na promoção da saúde na atenção primária, Costa et al. ${ }^{22}$ descrevem que ao longo da história do SUS foram criadas diversas estratégias para efetivar a assistência farmacêutica como uma política pública baseada no princípio da integralidade. 0 trabalho tradicional - preparação e dispensação de medicamentos mostrou-se insuficiente para definir a assistência farmacêutica no SUS ${ }^{23}$. Corroborando tal observação, estudo ${ }^{24}$ apontou a importância desse tema diante da complexidade nacional, das mudanças políticas, econômicas e sociais que impactaram nas políticas públicas e no acesso a medicamentos.

Sendo assim, a assistência farmacêutica se caracteriza como resultado da estrutura, pessoas e tecnologias, considerando o contexto social, com o objetivo principal de promover melhores condições de saúde para a população, com base em ações de promoção, prevenção, recuperação e reabilitação ${ }^{23}$. Acredita-se que o principal avanço da assistência farmacêutica no contexto do SUS seja a implementação da Política Nacional de Medicamentos e sua relação com o princípio da integralidade ${ }^{25}$, e, dessa maneira, em harmonia com o paradigma da promoção da saúde.

Para visualizar, entre os artigos analisados, o método utilizado (Figura 4), foi possível identificar que o método empregado com maior frequência foi o qualitativo, presente em 42 dos 123 artigos estudados, enquanto 33 artigos utilizaram o método quantitativo, e quatro utilizaram simultaneamente as duas abordagens.

A pesquisa qualitativa prevaleceu nos estudos sobre o tema da promoção da saúde na atenção primária. Para Oliveira et al. ${ }^{26}$, a pesquisa qualitativa se caracteriza por traduzir a realidade do objeto de estudo, sendo que esse conhecimento é capaz de modificar essa realidade investigada.

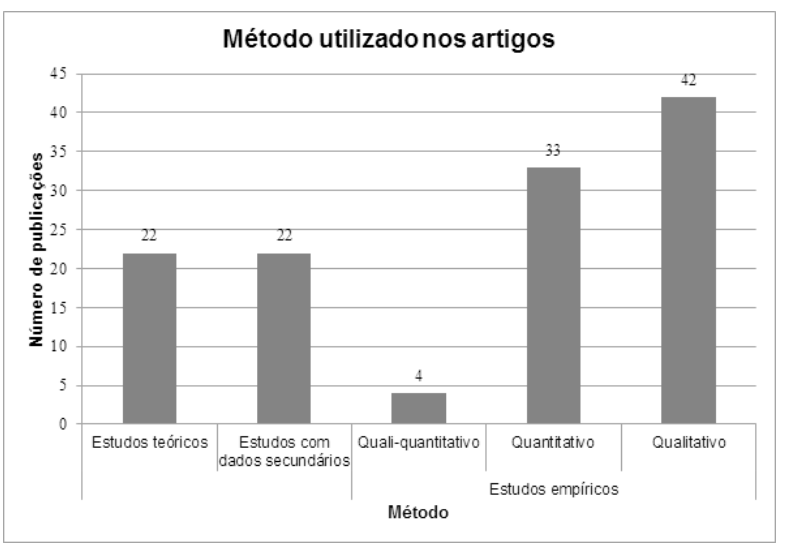

Figura 4. Método utilizado nas publicações

$(n=123)$.

Fonte: Dados da pesquisa.

No entanto, pesquisas que utilizaram as duas abordagens ainda foram incipientes nesse estudo bibliométrico. A combinação de estudos qualitativos e quantitativos é indicada quando o objeto de estudo necessita de uma compreensão mais ampla ${ }^{27}$. Nesse sentido, Santos et al. ${ }^{28}$ relataram que a pesquisa que utiliza métodos mistos tem por objetivo uma resposta ampliada a fenômeno de pesquisa, sendo uma alternativa para a investigação de questões complexas, que com frequência são enfrentadas pelo setor público de saúde.

Dos estudos empíricos $(n=79)$, a população que prevaleceu nas pesquisas (Figura 5) foi a dos profissionais de saúde $(n=36)$ e, em segundo lugar, os idosos $(n=10)$. Observou-se que poucos estudos $(n=6)$ utilizaram a combinação de duas ou mais populações (amostra) distintas.

Os profissionais de saúde, de distintas categorias, foram os principais sujeitos nas pesquisas incluídas neste estudo bibliométrico. Brito et al. ${ }^{29}$, em pesquisa com o objetivo de investigar a percepção sobre o trabalho na Estratégia Saúde da Família, encontraram como principais achados que, embora as ações de promoção da saúde tenham sido citadas pelos profissionais, os resultados indicaram que eles ainda dispensam pouco tempo para as atividades baseadas na promoção da saúde. $0 s$ mesmos autores ${ }^{29}$ sugerem a necessidade de qualificação tanto do ensino em saúde quanto da educação permanente dos profissionais de saúde inseridos na atenção primária. 


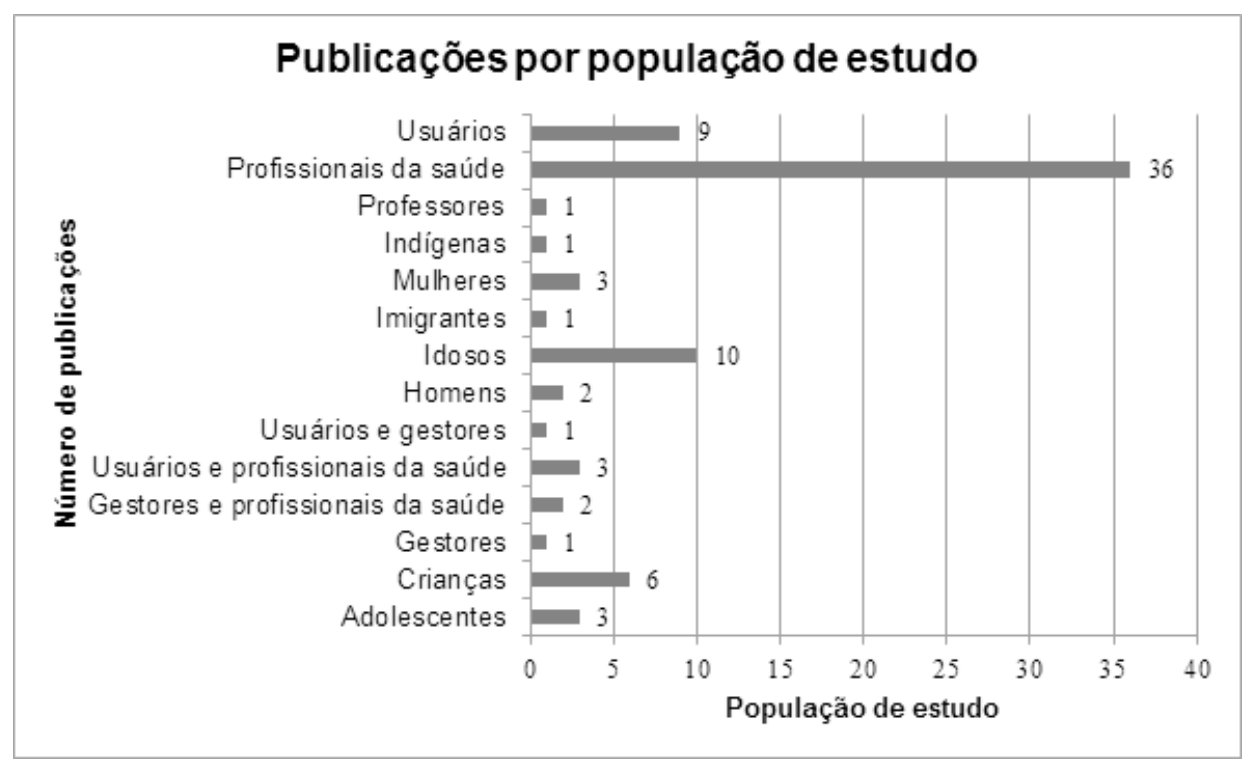

Figura 5. Publicações por população de estudo nas pesquisas empíricas $(n=79)$. Fonte: Dados da pesquisa.

Em estudo ${ }^{30}$ com o objetivo de compreender as experiências de organização dos sistemas públicos de saúde em relação à promoção da saúde em unidades de atenção primária de Florianópolis, Santa Catarina, destacaram que a atenção à saúde ainda se mostra baseada no modelo preventivo, devido a uma formação voltada ao modelo biomédico dos profissionais. Ainda, ressalta-se o imperativo de ampliar a compreensão acerca das ações de promoção da saúde, que devem ser articuladas com os determinantes de saúde da população adstrita.

A segunda população mais pesquisada nos estudos foi a dos idosos. Diante disso, existe a necessidade urgente de promover a saúde dos idosos com ambientes saudáveis e evitando os fatores de risco que podem ser modificados.

Para a saúde do idoso, a PNPS aponta especial relevância às ações voltadas ao processo de envelhecimento ${ }^{7}$. Complementando, Araújo et al. ${ }^{31}$ descreveram que é importante que o sistema de saúde se organize frente ao envelhecimento, com destaque para o trabalho com os grupos de convivência para os idosos na atenção primária como estratégia de promoção da saúde dessa população.

Por fim, a análise bibliométrica dos 123 artigos permitiu que fosse possivel obter um panorama da produção acadêmica nacional através da base indexadora SciELO, de modo a direcionar as lacunas existentes na produção e consequente evolução da temática da promoção da saúde na atenção primária, no período de 2006 a novembro de 2019.

\section{CONCLUSÃO}

A pesquisa bibliométrica evidenciou um maior número de publicações nos anos de 2017-2016 e nas Revistas Ciência \& Saúde Coletiva e Saúde Pública, respectivamente. Com a mudança do perfil demográfico e epidemiológico da população brasileira, este estudo destaca a importância de pesquisas sobre ações de promoção da saúde na atenção primária, fomentando o aumento de publicações sobre a temática nos próximos anos.

0 principal achado desta pesquisa se reporta aos assuntos principais sobre as publicações relacionadas à promoção da saúde na atenção primária, que foram a alimentação/nutrição e assistência farmacêutica. Realça-se que os estudos envolveram distintos assuntos, descrevendo a transversalidade da promoção da saúde na atenção primária, sendo caracterizada como intersetorial, interdisciplinar e multiprofissional.

0 método mais utilizado nos estudos foi 0 empírico, com a pesquisa qualitativa. Sendo que, na pesquisa empírica, a população de estudo que predominou foi a de profissionais da saúde e, em segundo lugar, de idosos. Destaca-se, aqui, a importância da triangulação de dados, combinando diferentes métodos de coleta e distintas amostras para compreender o objeto de estudo de maneira ampla e profunda.

Como limitações do estudo, pode-se citar a pesquisa ter sido realizada em apenas uma base de dados, o que não permite um panorama geral sobre as 
publicações oriundas de pesquisadores brasileiros, visto que se busca pela produção internacional como tentativa de reconhecimento sobre as pesquisas desenvolvidas no contexto brasileiro. Recomendam-se estudos com outras variáveis de análise utilizadas na pesquisa bibliométrica, bem como incluir estudos de abrangência internacional.

\section{CONTRIBUIÇÃO DAS AUTORAS}

Xavéle Braatz Petermann contribuiu com o delineamento, a realização da pesquisa e a redação do manuscrito. Sheila Kocourek contribuiu com o delineamento da pesquisa e a revisão crítica do manuscrito.

\section{REFERÊNCIAS}

1. Buss PM, Carvalho AI. Desenvolvimento da promoção da saúde no Brasil nos últimos vinte anos (1988-2008). Ciênc Saúde Colet. 2009;14(6):2305-16. doi: https://doi.org/10. 1590/S1413-81232009000600039

2. Pereira IC, Oliveira MA. Atenção primária, promoção da saúde e o Sistema Único de Saúde: um diálogo necessário. São Paulo: Escola de Enfermagem da Universidade de São Paulo; 2014.

3. World Health Organization. Declaration of Alma-Ata International Conference on Primary Health Care, Alma-Ata, USRR, 6-12 Sept. 1978 [document on the internet]. Geneve; 1978 [cited 2019 July 07]. Available from: https:// www.who.int/publications/almaata declaration en.pdf

4. World Health Organization. The Ottawa Charter for Health Promotion. First International Conference on Health Promotion, Ottawa, 21 Nov. 1986 [document on the internet]. Geneve; 1986 [cited 2019 July 07]. Available from: https:// www.who.int/teams/health-promotion/enhancedwellbeing/first-global-conference.

5. Aith FM. Marcos legais da promoção da saúde no Brasil. Rev Med. 2013;92(2):148-54. doi: https:// doi.org/10.11606/issn.1679-9836.v92i2p148-154

6. Silva PF, Baptista TW. Os sentidos e disputas na construção da Política Nacional de Promoção da Saúde. Physis. 2014;24(2):441-65. doi: https:// doi.org/10.1590/S0103-73312014000200007

7. Brasil. Política Nacional de Promoção da Saúde: PNaPS: revisão da Portaria MS/GM n 687, de 30 de março de 2006 [document on the internet]. Brasilia (DF): Ministério da Saúde; 2014 [cited 2019 July 07]. Available from: http://bvsms.saude.gov.br/b vs/publicacoes/pnps revisao portaria 687.pdf
8. Brasil. Portaria GM/MS $n^{\circ} 687$, de 30 de março de 2006, que institui a Política Nacional de Promoção da Saúde [document on the internet]. Brasília (DF): Ministério da Saúde; 2014 [cited 2019 July 07]. Available from: https://bvsms.saude.gov.br/bvs/saudelegis/ $\mathrm{gm} / 2006 /$ prt0687 30 03 2006.html

9. Malta DC, Morais Neto OL, Silva MM, Rocha D, Castro $A M$, Reis AA, et al. Política Nacional de Promoção da Saúde (PNPS): capítulos de uma caminhada ainda em construção. Ciênc Saúde Colet. 2016;21(6):1683-94. doi: https://doi.org/10.1590/1413-81232015216. $\underline{07572016}$

10. Dias MS, Oliveira IP, Silva LM, Vasconcelos MI, Machado MD, Forte FD, et al. Política Nacional de Promoção da Saúde: um estudo de avaliabilidade em uma região de saúde no Brasil. Ciênc. Saúde Colet. 2018; 23(1):103-14. doi: https://doi. org/10.1590/1413-81232018231.24682015

11. Chueke GV, Amatucci M. 0 que é bibliometria? Uma introdução ao Fórum. Internext [serial on the internet]. 2015 [cited 2019 July 07];10(2):15. Available from: https://internext.espm.br/ internext/article/view/330/233

12. Melo Ribeiro HC. Bibliometria: quinze anos de análise da produção acadêmica em periódicos brasileiros. Biblios. 2017;69:120. doi: http://dx.doi.org/10.5195/biblios. $\underline{2017.393}$

13. Moura LKB, Azevedo UN, Wingwrter DG, Ferreira MA, Maciel MP, Moura RP, et al. Análise bibliométrica das evidências científicas sobre violência contra a pessoa idosa. Ciênc Saúde Colet. $2020 ; 25(6): 2143$ 52. doi: https://doi.org/10.1590/1413-8123202 $\underline{0256.226322018}$

14. Sobre o Scielo. Scientific Electronic Library Online [home-page on the internet]. [cited 2019 Dec 11]. Available from: https://www.scielo.org/ pt/sobre-o-scielo

15. Paim JS. Sistema Único de Saúde (SUS) aos 30 anos. Ciênc Saúde Colet. 2018 Jun;23(6): 1723-28. doi: https://doi.org/10.1590/1413$\underline{81232018236.09172018}$

16. Prado NMBL, Santos AM. Promoção da saúde na Atenção Primária à Saúde: sistematização de desafios e estratégias intersetoriais. Saúde debate. 2018;42(1):379-95. doi: https://doi. org/10.1590/0103-11042018S126

17. Malta $D C$, Reis $A A$, Jaime $P C$, Morais Neto, Silva MM, Akerman M, et al. O SUS e a Política Nacional de Promoção da Saúde: perspectiva resultados, avanços e desafios em tempos de crise. Ciênc Saúde Colet. 2018;23(6):1799-1809. doi: https://doi. org/10.1590/1413-81232018236.04782018 
18. Sobre a Revista Ciência e Saúde Coletiva [homepage on the internet]. Ciênc Saúde Colet [data desconhecida]. [cited $2019 \mathrm{Dec} 11$ ]. Available from: https://www.scielo.br/journal/csc/about/\#about -

19. Sobre a Revista de Saúde Pública [home-page on the internet]. Rev Saúde Públ [data desconhecida]. [cited 2019 Dec 11]. Available from: https://www. scielo.br/journal/rsp/about/\#about -

20. Jaime PC, Delmuè DC, Campello T, Silva D0, Santos LM. Um olhar sobre a agenda de alimentação e nutrição nos trinta anos do Sistema Único de Saúde. Ciênc Saúde Colet. 2018;23(6):182936. doi: https://doi.org/10.1590/1413$\underline{81232018236.05392018}$

21. Magalhães, R. Avaliação da Política Nacional de Promoção da Saúde: perspectivas e desafios. Ciênc Saúde Colet. 2016;21(6):1767-76. doi: https://doi. org/10.1590/1413-81232015216.07422016

22. Costa EA, Araújo PS, Penaforte TR, Barreto JL, Guerra Junior AA, Acurcio FA, et al. Concepções de assistência farmacêutica na atenção primária à saúde no Brasil. Rev Saúde Pública. 2017;51(suppl.2). doi: https://doi.org/10.11606/ $\underline{\text { S1518-8787.2017051007107 }}$

23. Brasil. Assistência farmacêutica na atenção básica: instruções técnicas para sua organização. 2. ed. Brasília (DF): Ministério da Saúde; 2006.

24. Bermudez JAZ, Esher A, 0sorio-de-Castro CG, Vasconcelos DM, Chaves GC, Oliveira MA, et al. Assistência Farmacêutica nos 30 anos do SUS na perspectiva da integralidade. Ciênc Saúde Colet. 2018;23(6):1937-49. doi: https://doi. org/10.1590/1413-81232018236.09022018

25. Brasil. Política Nacional de Medicamentos. Brasília (DF): Ministério da Saúde; 2001.

26. Oliveira ES, Costa AP. Pesquisa qualitativa: desenvolvimento e perspectivas no campo da promoção da saúde. Rev bras promoç saúde [serial on the internet]. 2016 [cited 2021 Jan 10];29(Supl):14. Available from: https://periodicos.unifor.br/ RBPS/article/view/6418/pdf

27. Lorenzini, E. Pesquisa de métodos mistos nas ciências da saúde. Rev cuid. 2017;8(2):1549-60. doi: https://doi.org/10.15649/cuidarte.v8i2.406

28. Santos JLG, Erdmann AL, Meirelles BH, Lanzoni $G M$, Cunha VP, Ross R. Integração entre dados quantitativos e qualitativos em uma pesquisa de métodos mistos. Texto Contexto Enferm. 2017;26(3). doi: https://doi.org/10.1590/0104$\underline{07072017001590016}$
29. Brito GEG, Mendes ACG, Santos Neto PM. 0 trabalho na estratégia saúde da família e a persistência das práticas curativistas. Trab educ saúde (Online). 2018;16(3):975-95. doi: https:// doi.org/10.1590/1981-7746-sol00164

30. Heidemann ITSB et al. Estudo comparativo de práticas de promoção da saúde na atenção primária em Florianópolis, Santa Catarina, Brasil e Toronto, Ontário, Canadá. Cad Saúde Pública. 2018;34(4):e00214516. doi: https://doi. org/10.1590/0102-311X00214516

31. Araújo LSA, Cypriano CC, Gastaldo D, Jackson S, Rocha CG, Fagundes E. Idosos e grupos de convivência: motivos para a não adesão. Sanare (Sobral, Online) [serial on the internet]. 2017 [cited 2021 Jan 10];16(1):58-67. Available from: https://sanare.emnuvens.com. $\mathrm{br} / \mathrm{sanare} /$ article/view/1140/625
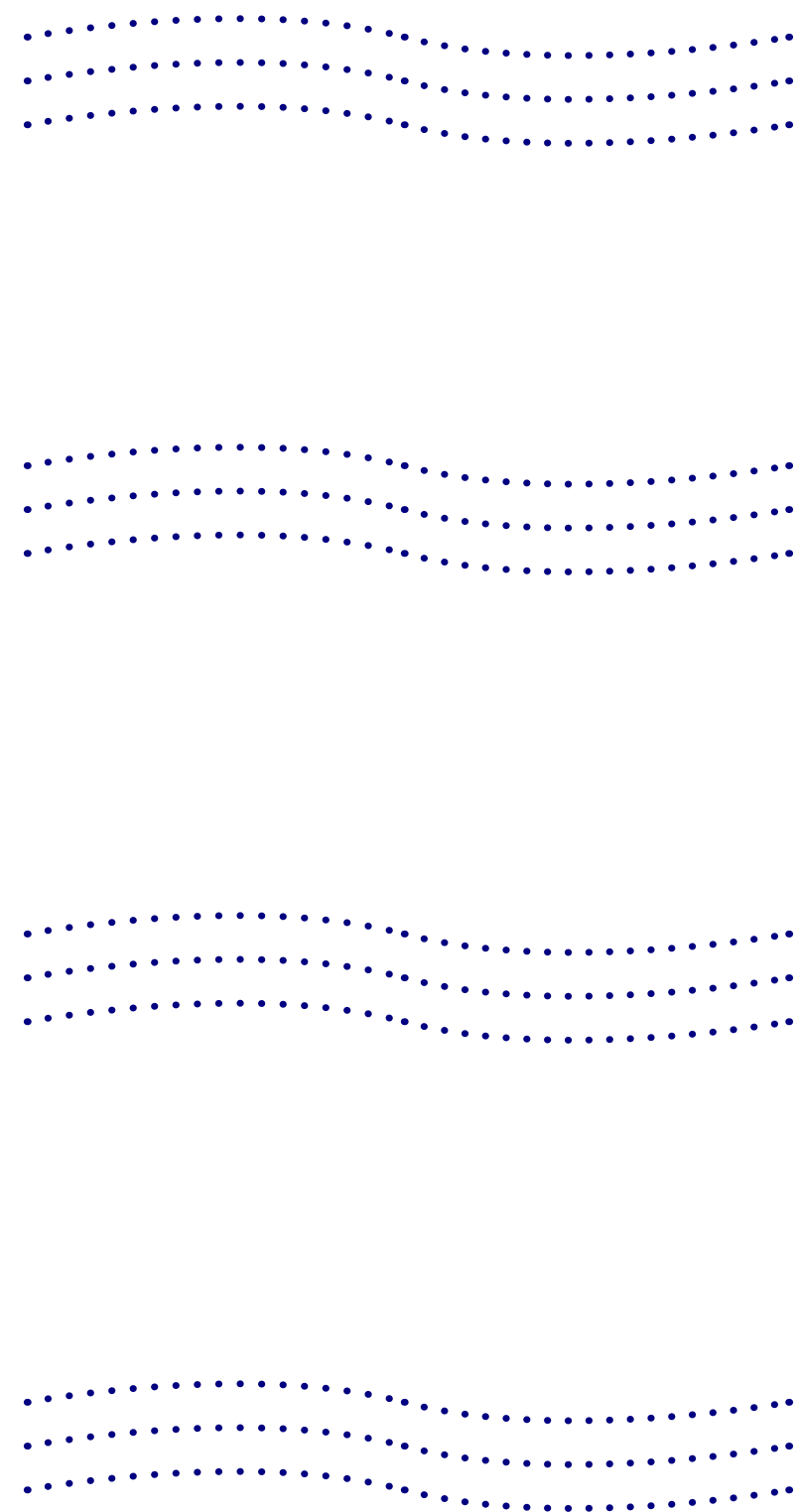